\title{
Prospective study of mycobacterial infections in patients with cystic fibrosis
}

Lena Hjelte, Björn Petrini, Gunilla Källenius, Birgitta Strandvik

\begin{abstract}
Fifty four patients with cystic fibrosis, aged 3-67 years, were studied prospectively for pulmonary mycobacterial infection. Sputum smears and cultures were carried out and intradermal skin tests performed. Mycobacteria were cultured from six patients in association with clinical deterioration; four patients had positive direct smears. Mycobacterium tuberculosis, $M$ aviumintracellulare, $M$ kansasii, and $M$ gordonae were isolated. There were no deaths and all improved with chemotherapy. A third of the other 48 patients had positive skin test responses $(>6 \mathrm{~mm}$ ) to purified protein derivative (PPD) tuberculin and 21 to one or more antigens prepared from non-tuberculous mycobacteria. Sensitisation increased with age; before the age of 11 only one patient had a positive response to $P P D$ tuberculin and none to any other antigen. This was less than in healthy control subjects of similar age. After age 11 the reactions in sensitised patients were stronger than in positive healthy control subjects. Our study indicates that it is important to consider mycobacterial infection in patients with cystic fibrosis who deteriorate without obvious cause.
\end{abstract}

In patients with cystic fibrosis the major cause of death is the progressive pulmonary disease with infections caused by Staphylococcus aureus, Haemophilus influenzae, and Pseudomonas species. Pulmonary infection with mycobacteria is a rare but potentially serious complication. ${ }^{1-3}$ We reported a three year prospective study using sputum culture and skin tests for mycobacterial infection in patients with cystic fibrosis.

\section{Methods}

PATIENTS

We investigated 54 patients with cystic fibrosis who attended the Stockholm cystic fibrosis centre regularly. All had previously been vaccinated with BCG, and all lived in the metropolitan area. Ten patients had contact with domestic animals or horses and two had contact with birds (cases 4 and 5). The mean age of the patients was 18 years (range 3-67) years (mean 19 years for the 23 males and 18 for the 31 females). The mean Shwachmann score was 78 (range 30-100). ${ }^{4}$ All patients had an abnormal sweat test result (chloride $(>80 \mathrm{mmol} / \mathrm{l}$ ) and pulmonary and gastrointestinal symptoms and all required treatment with pancreatic supplements, mucolytic agents (including by inhalation), and physiotherapy. Thirty one patients were chronically colonised with Pseudomonas aeruginosa or other Pseudomonas species and 13 with $S$ aureus in addition. Ten patients were colonised only with $S$ aureus. Six patients had diabetes mellitus. One patient required prednisolone because of severe asthma.

SKIN TESTS

The following antigens were used for intradermal injections: PPD-T (PPD tuberculin in Tween diluent, $2 \mathrm{TU} / 0.1 \mathrm{ml}$ ), PPD-A ( $M$ avium antigen RS 10, $0.1 \mu \mathrm{g} /$ $0.1 \mathrm{ml})$, PPD-I ( $M$ intracellulare antigen RS $0 \cdot 1 \mu \mathrm{g} / 0 \cdot 1 \mathrm{ml}$ ), PPD-S (M scrofulaceum antigen RS 95, $0.1 \mu \mathrm{g} / 0.1 \mathrm{ml})$, PPD-K (M kansasii, RS 30, $0 \cdot 1 \mu \mathrm{g} / 0 \cdot 1 \mathrm{ml})$. All antigens were obtained from Statens Seruminstitut, tuberculin department, DK-2300 Copenhagen $S$, Denmark. Each antigen $(0.1 \mathrm{ml})$ was injected on the volar side of the middle third of the forearm and read at 72 hours by two specially trained nurses. A palpable induration of more than $6 \mathrm{~mm}$ diameter was regarded as positive.

\section{SPUTUM ANALYSES}

At least three direct smear examinations and three cultures for mycobacteria were performed for each patient except for eight patients not producing sputum. Gastric lavage was performed in six patients, five of whom had one or more strongly positive skin test responses. Smears were examined after being stained with auramine. Mycobacterial isolates were identified by standard biochemical methods. ${ }^{5}$ Susceptibility to streptomycin, amikacin, isoniazid, ethambutol and rifampicin was determined on Löwenstein-Jensen medium by the resistance ratio method. ${ }^{6}$ Routine laboratory haematological determinations, serum immunoglobulin measurements, and liver and renal function tests were performed. Spirometric values were compared with those of healthy children and adults. ${ }^{78}$

\section{STATISTICS}

Statistical analyses were performed by means of the $\chi^{2}$ test. 
Table 1 Bacteriological findings and skin test results in six patients with cystic fibrosis and positive sputum cultures of mycobacteria

\begin{tabular}{|c|c|c|c|c|c|c|c|c|c|c|c|}
\hline \multirow[b]{2}{*}{ Patient No } & \multirow[b]{2}{*}{ Sex } & \multirow[b]{2}{*}{ Age (y) } & \multicolumn{2}{|c|}{$\begin{array}{l}\text { Chronic sputum } \\
\text { colonisation }\end{array}$} & \multirow[b]{2}{*}{ Direct smear ${ }^{\star}$} & \multirow[b]{2}{*}{ Mycobacteria in sputum } & \multicolumn{5}{|c|}{$P P D \dagger$ skin test results ( $\mathrm{mm}$ ) } \\
\hline & & & Psa & $S a$ & & & $T$ & $A$ & $I$ & $s$ & $\boldsymbol{K}$ \\
\hline 1 & $\mathbf{F}$ & 11 & + & + & + & $M$ avium-intracellulare & $\begin{array}{c}0 \\
14 \ddagger\end{array}$ & $\begin{array}{l}22 \\
16\end{array}$ & $\begin{array}{l}17 \\
\text { ND }\end{array}$ & $\begin{array}{l}18 \\
\text { ND }\end{array}$ & $\begin{array}{l}\text { ND } \\
15\end{array}$ \\
\hline 2 & $\mathrm{~F}$ & 19 & + & + & + & $\begin{array}{l}M \text { kansasii } \\
M \text { avium-intracellulare }\end{array}$ & $\begin{array}{l}10^{+} \\
15 \S\end{array}$ & $\begin{array}{l}10 \\
13\end{array}$ & $\begin{array}{l}6 \\
\text { ND }\end{array}$ & $\begin{array}{l}8 \\
\text { ND }\end{array}$ & $\begin{array}{l}\text { ND } \\
11\end{array}$ \\
\hline 3 & $\mathbf{F}$ & 16 & + & - & + & Unclassified mycobacteria & 19 & 15 & 9 & 11 & ND \\
\hline 4 & $M$ & 16 & + & + & - & M gordonae & 0 & 0 & 0 & 0 & ND \\
\hline 5 & $\mathbf{F}$ & 25 & - & + & - & $M$ tuberculosis & 13 & 0 & 0 & 0 & ND \\
\hline 6 & $\mathrm{~F}$ & 34 & + & - & + & $M$ avium-intracellulare & 11 & 18 & 13 & 16 & ND \\
\hline
\end{tabular}

*Positive $(+)$ or negative $(-)$ for mycobacteria.

+Purified protein derivatives (PPD): T-PPD-tuberculin; A-PPD-avium; I-PPD-intracellulare; S-PPD-scrofulaceum; K-PPD-kansasii.

+ Six months after the first investigation.

Eighteen months after the first investigation.

Psa-Pseudomonas aeruginosa; Sa-Staphylococcus aureus; ND-not done.

Results of yearly spirometry in six patients with cystic.fibrosis in whom mycobacteria were isolated in sputum at the time of clinical

deterioration (indicated by arrow).VC-vital capacity; $F E V_{1}-$ forced expiratory volume in one second. $\square$ Patient 1; $\square 2$; $\triangle 3 ; \triangle 4 ; 05 ; 06$.

\section{Results}

Sputum cultures grew $M$ tuberculosis, $M$ avium-intracellulare, $M$ kansasii and $M$ gordonae in six patients (table 1). All six patients had received BCG vaccination in the first weeks of life. At the time of diagnosis they had deteriorated clinically with weight loss, considerable progression of the shadowing on chest radiographs, and impairment of lung function (figure). They reported intermittent fever, and patient 6 , the only one with lymphadenopathy, had nightly attacks of profound sweating. All patients improved with specific chemotherapy (streptomycin, rifampicin, isoniazid, and ethambutol in standard doses). No case was fatal and no contacts were identified.

The skin test results in the patients from whom mycobacteria were isolated from sputum are given in table 1. Four patients had positive direct smears. All but one (No 4) had at least

\section{$\%$ \\ predicted}

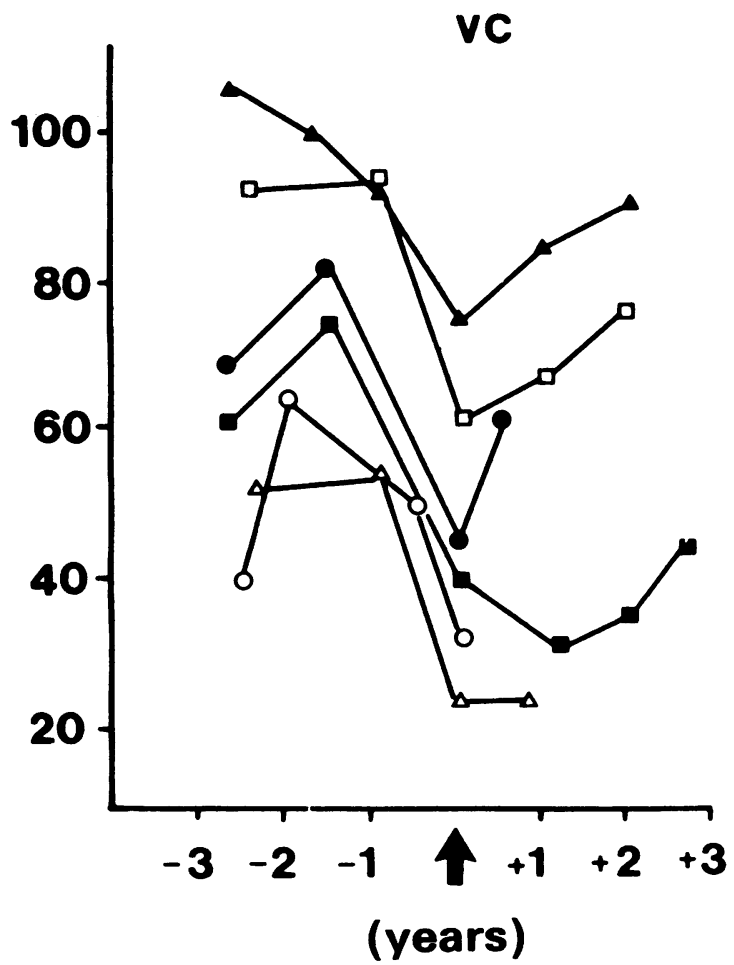

two positive sputum cultures. In one patient (No 2) two species of mycobacteria were isolated within 18 months. The largest skin test reaction corresponded to the mycobacterial species isolated in three patients (four instances).

The results of skin tests in the other patients with cystic fibrosis are given in table 2 . One third of the patients had a positive response to PPD tuberculin and almost half to one or more antigens from non-tuberculous mycobacteria. Fifteen of the 21 patients who had a positive response to one or more non-tuberculous antigens had a positive response to PPD tuberculin. Only two patients had a positive response to PPD tuberculin and a negative response to all other antigens; one had had BCG vaccination 18 months before. Of those with a positive response to any non-tuberculous antigen, 13 had a positive response to three of the four antigens, seven to two, and two to one.

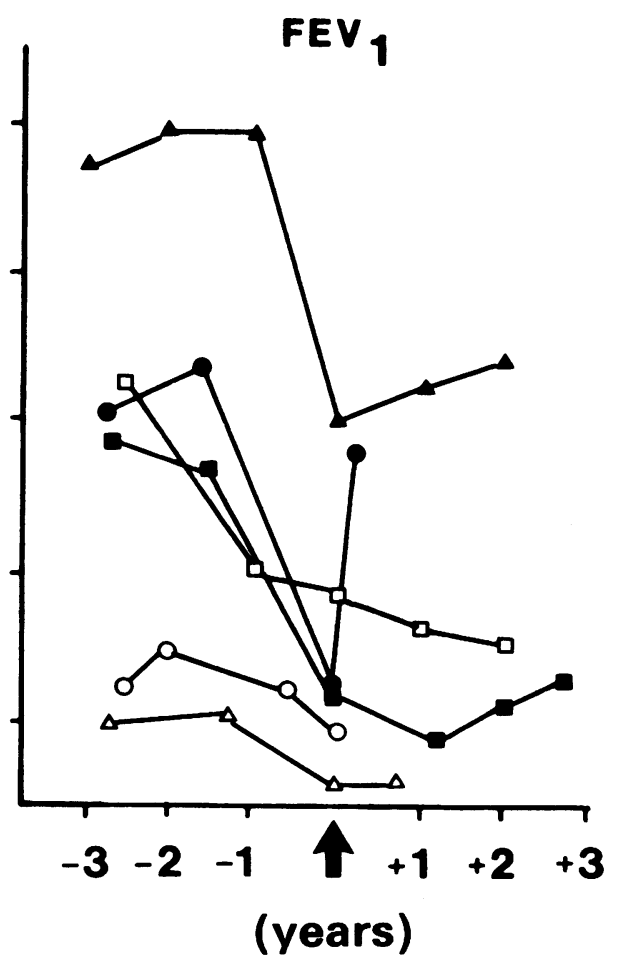


Table 2 Numbers of patients with cystic fibrosis and sputum cultures negative for mycobacteria who had positive responses ( $>6 \mathrm{~mm}$ ) to skin tests with mycobacterial antigens, by age

\begin{tabular}{ccc}
\hline Age $(y)$ & $P P D-T$ & Other antigens* \\
\hline $0-10$ & $1 / 11$ & $0 / 11$ \\
$11-15$ & $2 / 10$ & $4 / 10$ \\
$16-20$ & $3 / 12$ & $8 / 12$ \\
$>21$ & $10 / 15$ & $9 / 15$ \\
All & $16 / 48\left(33^{\circ}{ }_{0}\right)$ & $21 / 48\left(44^{\circ}{ }_{0}\right)$ \\
\hline
\end{tabular}

‡One or more of PPD-A, PPD-I, and PPD-S (see second footnote to table 1 )

Only one of 11 patients under the age of 11 had a positive response to any antigen (PPD tuberculin in the one case) despite prior BCG vaccination. Sensitisation to antigens increased with age (table 2).

Positive skin test responses to PPD-tuberculin occurred in $20^{\circ}$ of patients aged 11-20 years with negative sputum cultures, compared with $44^{\circ}{ }_{0}$ of healthy 14 year old Swedish children who had BCG vaccination at birth ${ }^{9}$ $(p<0.05)$ and half of 8-9 year old Swedish children vaccinated with $\mathrm{BCG}(\mathrm{p}<0.05) .^{10}$ No patient reacted to any of the non-tuberculous antigens before the age of 11 , compared with $62-68^{\circ}{ }_{o}$ of healthy vaccinated 8-9 year old children $^{10}(\mathrm{p}<0.001)$. A difference in the opposite direction was noticed when indurations of more than $10 \mathrm{~mm}$ were compared; these occurred in $38^{\circ}{ }_{0} \quad(M$ aviumintracellulare) and $31^{\circ}{ }_{0}$ (M scrofulaceum) of patients with cystic fibrosis (data not shown) and in $10^{\circ}{ }_{0}$ and $13^{\circ}{ }_{0}$ of healthy children ${ }^{10}$ ( $p<0.001$ and $p<0.001$ respectively).

No relation was found between skin test results and erythrocyte sedimentation rate, total white blood cell count, differential white cell count, serum IgG, clinical state, $\mathrm{FEV}_{1}$, or chest radiographs (data not shown). Only two of the patients with positive mycobacterial cultures were in a poor nutritional state ( $<2$ SD for weight), and one was receiving corticosteroid treatment; none had diabetes mellitus.

\section{Discussion}

Colonisation by non-tuberculous mycobacteria may be facilitated by bronchiectasis and cavitary disease. ${ }^{11}$ It is surprising therefore that mycobacteria are isolated so infrequently from patients with cystic fibrosis. ${ }^{1}$ Previous reported cases have usually been fatal. ${ }^{23} \mathrm{~A}$ report of 16 years' experience at the Brompton Hospital, London, showed that during the first 10 years no mycobacteria were isolated. ${ }^{12}$ In the six years after the introduction of routine direct smears and culture of sputum for mycobacteria in patients with cystic fibrosis seven of 223 patients were found to have positive sputum samples. In our screening of patients one proved to have tuberculosis, three had nontuberculous mycobacterial infection, and two had equivocal infection (patients 3 and 4; table 1).

The relevance of mycobacteria isolated from sputum may be difficult to assess, especially in patients with cystic fibrosis or other chronic pulmonary diseases. We found both an obligate pathogen ( $M$ tuberculosis) and one isolate ( $M$ gordonae) that is rarely associated with disease. ${ }^{1314} M$ avium-intracellulare and $M$ kansasii form an intermediate group, causing either progressive lung disease or colonisation only. ${ }^{15}$ The deterioration in lung function observed in all patients at the time of positive sputum culture suggests that these mycobacteria were causing disease (figure). Furthermore, infection is more probable than colonisation in cases with positive direct smears because these indicate a large number of mycobacteria in the sputum. Such findings with repeated isolation of the same bacterial strain and skin reactivity to the relevant antigen may be helpful for diagnosis. ${ }^{1617}$ As mycobacteria are known to possess cross reacting antigens, various PPD preparations should be used for comparison and chosen according to geographical distribution. ${ }^{18}$ Our patients seemed to be less sensitised to non-tuberculous antigens than healthy young people in Sweden, but when positive the reaction seemed to be stronger than that in the healthy population. Our finding of a lower rate of sensitisation in patients with cystic fibrosis than in healthy children seems to be age related and might be related to biochemical changes during the progression of the disease. One progressive disturbance in cystic fibrosis is deficiency of essential fatty acids ${ }^{19}$ and abnormal eicosanoid production (Strandvik et al, unpublished observations). Interestingly, administration of indomethacin, in vitro and in vivo, reverses subnormal immune reactivity to non-tuberculin mycobacterial antigens,${ }^{20}$ because patients with cystic fibrosis overproduce some eicosanoids. ${ }^{21} \mathrm{~T}$ cell function has been considered to be normal in patients with cystic fibrosis, ${ }^{22}$ but further studies are needed.

Our study indicates that mycobacterial infection should be considered in patients with cystic fibrosis who deteriorate without known cause. Sputum examination must be performed because skin tests are of limited value. In contrast to some earlier reports, none of our cases was fatal, indicating that chemotherapy may be life saving in non-tuberculous mycobacterial infection.

The skilful technical assistance of Irén Ladányi and Berit Widén is gratefully acknowledged.

1 Wood RE, Boat TF, Doershuk CF. Cystic fibrosis. Am Rev Respir Dis 1976;113:833-77.

2 Boxerbaum B. Isolation of rapidly growing mycobacteria in patients with cystic fibrosis. J Pediatr 1980;96:689-91.

3 Efthimiou J, Smith MJ, Hudson M, Batten JC. Fatal pulmonary infection with Mycobacterium fortuitum in cystic fibrosis. Br J Dis Chest 1984;78:299-302.

4 Shwachmann $\mathrm{H}$, Kulczycki LL. Long term study of onehundred-and-five patients with cystic fibrosis. Am J Dis Child 1958;96:6-15.

5 Vestal AL. Procedures for the isolation and identification of mycobacteria. Atlanta: Centers for Disease Control, 1979.

6 Canetti G, Froman S, Grosset J, et al. Mycobacteria: laboratory methods for testing drug sensitivity and resislaboratory methods for testing drug
tance. Bull WHO 1963;29:565-78.

7 Engström J, Karlberg P, Swarts CL. Respiratory studies in children. IX Relationships studies in children properties of lung, lung volumes and ventilatory capacity in healthy of lung, lung volumes and ventilatory capacity in healthy
children 7-15 years of age. Acta Paediar Scand 1962;51:68-86. 
8 Berglund E, Birath G, Bjure J, et al. Spirometric studies in normal subjects. I. Forced expirograms in subjects between 7 and 70 years of age. Acta Med Scand 1963;173:185-92.

9 Beskow R, Bleiker M, Dahlström G, Mellvin R, Sjögren I, Styblo $\mathrm{K}$. Tuberculin sensitivity in Swedish school children vaccinated at birth [Swedish]. Läkartidningen $1981 ; 78: 118-20$

10 Lind A, Bentzon MW, Doshé I-M, et al Sensitivity to tuberculin and sensitins in Swedish children. Bull Int Union Tuberc Lung Dis 1988;63:19-22.

11 Tsukamura $M$. Background factors for casual isolation of Mycobacterium intracellulare from sputum of patients with Mycobacterium intracellulare from sputum of patients

12 Smith MJ, Efthimiou J, Hodson ME, Batten JC. Mycobacterial isolations in young adults with cystic

13 Woods GL, Washington JA 2d. Mycobacteria other than Mycobacterium tuberculosis: review of microbiologic and clinical aspects. Rev Infect Dis 1987;9:275-94.

14 Chan J, McKitrick JC, Klein RS. Mycobacteriumgordonae in the aquired immunodeficiency syndrome [letter]. Ann Intern Med 1984;101:400.

15 Ahn CH, McLarty JW, Ahn SS, Ahn SI, Hurst GA Diagnostic criteria for pulmonary disease caused by Mycobacterium kansasii and Mycobacterium intracellulare. Am Rev Respir Dis 1982;125:388-91.
16 Margileth AM. The use of purified protein derivative mycobacterial skin test antigens in children and adolescents; purified protein skin test results correlated with mycobacterial isolates Pediatr Infect Dis 1983;2:225-31.

17 Marmorstein BL, Sheinhorn DJ. The role of nontuberculous mycobacterial skin test antigens in the diagnosis of mycobacteria infections. Chest 1975;67:320-4.

18 Chaparas SD. Mycobacterial antigens: immunologically based diagnostic tests with tuberculin and other mycobacterial antigens. New York: Dekker, 1984:195-220.

19 Rogiers V, Vercruysse A, Dab I, Baran D. Abnormal fatty acid pattern of the plasma cholesterol ester fraction in cystic fibrosis patients with and without pancreatic insufficiency. Eur J Pediatr 1983;141:39-42.

20 Mason VG III, Kirkpatrick CH. Modulation of immunologic responses in nontuberculous mycobacterial infeclogic responses in nontuberculous mycobacterial infec-

21 Zakrzewski JT, Barnes NC, Piper PJ, Costello JF. Detection of sputum eicosanoids in cystic fibrosis and in normal saliva by bioassay and radioimmunoassay. $\mathrm{Br} J \mathrm{Clin}$ Pharmacol 1987;23:19-27.

2 Carlastedt-Duke J, Brönnegård $M$, Strandvik B. Pathological regulation of archidonic acid release in cystic fibrosis: The putative basic defect. Proc Natl Acad Sci USA 1986;83:9202-6. 